\title{
A Novel Role for Programmed Cell Death Receptor Ligand-1 in Sepsis-Induced Intestinal Dysfunction
}

\author{
Youping Wu, ${ }^{1 *}$ Chun-Shiang Chung, ${ }^{2 *}$ Yaping Chen, ${ }^{2}$ Sean Farrell Monaghan, ${ }^{2}$ Sima Patel, ${ }^{3}$ Xin Huang, ${ }^{2}$ \\ Daithi Seamus Heffernan, ${ }^{2}$ and Alfred Ayala ${ }^{2}$
}

${ }^{1}$ Department of Anesthesiology, Changhai Hospital, Second Military Medical University, Shanghai, People's Republic of China; ${ }^{2}$ Department of Surgery, Division of Surgical Research, Alpert School of Medicine at Brown University/Rhode Island Hospital, Providence, RI, United States of America; and ${ }^{3}$ Department of Biochemistry and Molecular Biology, Brown University, Providence, RI, United States of America

\begin{abstract}
Studies imply that intestinal barrier dysfunction is a key contributor to morbid events associated with sepsis. Recently, the co-inhibitory molecule programmed death-ligand1 (PD-L1) has been shown to be involved in the regulation of intestinal immune tolerance and/or inflammation. Our previous studies showed that PD-L1 gene deficiency reduced sepsis-induced intestinal injury morphologically. However, it is not known how PD-L1 expression impacts intestinal barrier dysfunction during sepsis. Here we tested the hypothesis that PD-L1 expressed on intestinal epithelial cells (IECs) has a role in sepsis-induced intestinal barrier dysfunction. To address this, C57BL/6 or PD-L1 gene knockout mice were subjected to experimental sepsis and PD-L1 expression, intestinal permeability and tissue cytokine levels were assessed. Subsequently, septic or nonseptic colonic samples (assigned by pathology report) were immunohistochemically stained for PD-L1 in a blinded fashion. Finally, human Caco2 cells were used for in vitro studies. The results demonstrated that PD-L1 was constitutively expressed and sepsis significantly upregulates PD-L 1 in IECs from C57BL/6 mice. Concurrently, we observed increased PD-L1 expression in colon tissue samples from septic patients. PD-L1 gene deficiency reduced ileal permeability and tissue levels of IL-6, TNF- $\alpha$ and MCP-1, and prevented ileal tight junction protein loss compared with WT after sepsis. Comparatively, while Caco2 cell monolayers also responded to inflammatory cytokine stimulation with elevated PD-L1 expression, increased monolayer permeability and altered/decreased monolayer tight junction protein morphology/expression, these changes were reversed by PD-L1 blocking antibody. Together these data indicate that ligation of PD-L1 plays a novel role in mediating the pathophysiology of sepsis-induced intestinal barrier dysfunction.
\end{abstract}

Online address: http://www.molmed.org

doi: 10.2119/molmed.2016.00150

\section{INTRODUCTION}

Approximately 750,000 patients are diagnosed with sepsis each year in the United States, and it has been reported that the incidence level has been rising since the 1970s. With a mortality rate of nearly $30 \%$, sepsis remains a major health problem worldwide (1). Unfortunately, numerous treatments based on antiinflammatory or anticoagulant therapies have failed to provide a survival benefit in human clinical trials. Therefore, more information about the pathophysiology of this syndrome is needed if we are to better understand it and identify/develop novel clinical therapies. In this respect, studies have demonstrated that septic patients exhibited impaired immunity associated with sustained loss of important immune cells $(2,3)$. Several aberrations in leukocyte function have also been documented in septic patients,

${ }^{*} Y W$ and C-SC contributed equally to this work as first authors.

Address correspondence to Alfred Ayala, Division of Surgical Research, Aldrich 227, Rhode Island Hospital, 593 Eddy Street, Providence, RI 02903, USA. Phone: 401-444-5158; Fax: 401-444-3278; E-mail:AAyala@lifespan.org

Submitted June 16, 2016; Accepted for Publication October 14, 2016; Published Online (www.moldmed.org) October 25, 2016.

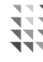

Feinstein Institute for Medical Research Northwell Health

which are associated with poor outcome (4-6). With the results of a small clinical trial showing that treatment with granulocyte-macrophage colony-stimulating factor could reverse sepsis-induced immune dysfunction (7), this suggests that strategies with immune adjuvant therapies might be of value.

It has been suggested that gut barrier dysfunction and/or increased intestinal permeability is a critical morbid event in the development of multiple organ failure during sepsis $(8,9)$. Increased permeability of the intestinal epithelium plays an important role in the pathophysiology of many gastrointestinal disorders, such as inflammatory bowel disease, irritable bowel syndrome, celiac disease and colon cancer (10), as well as in critical ill patients and experimental animals with multiple trauma, burn injury, hemorrhagic shock and sepsis (8). 
The intestinal mucosal immune system includes a variety of lymphoid compartments, which play a crucial role in the development and regulation of both innate and acquired immune defense systems (10). However, the mechanism of how gut dysfunction evolves during the septic process is not fully understood.

Recently, the coinhibitory protein programmed death-1 (PD-1 or CD279) and its ligand PD-L1 (B7-H1 or CD274) have been reported to be important in the regulation of immune function in animals and patients with sepsis. Previous studies have demonstrated that PD-L1 gene deficiency can protect mice from sepsis-induced organ injury and lethality $(11,12)$ and blockade of PD-1:PD-L1 ligation with antibody prevented the development of colitis in mice (13). Reports have also shown that treatment of immune cells derived from septic patients with PD-L1 antibodies decreased apoptosis and improved function (14). Additionally, studies have indicated that PD-L1 is not only expressed, but also involved in intestinal mucosal inflammation and regulates gut immune tolerance $(15,16)$. PD-L1 has been reported to be expressed on colonic and gastric epithelial cells, and in select situations it contributes to the interaction between epithelial cells and lymphocytes $(15,17,18)$. Intestinal epithelium is a key component of the intestinal barrier, which separates bacteria and toxic substances in the gut from the submucosal lymphoid tissue and maintains intestinal immune homeostasis. Tight junctions (TJs), located on the apical portion of the paracellular space between adjacent epithelial cells, are critical structures for the maintenance of effective barrier integrity in the epithelium and are major regulators of epithelial paracellular permeability. Experimentally, increased intestinal permeability can be induced by oxidant stress, hypoxia, nitric oxide and cytokines, which are all reported to be present during sepsis (9). While recent morphological studies suggest that expression of PD-L1 appears to contribute to sepsisinduced intestinal injury (12), it is unclear how this happens and whether this is the result of direct or indirect action through local expression of PD-L1. To address this, we examined the change in PD-L1 expression and its role in the intestinal mucosal immune response as well as its impact on gut barrier function in mice. To the extent such changes are also evident in humans, we compared PD-L1 expression in colonic tissues from septic and nonseptic patients.

\section{MATERIALS AND METHODS}

\section{Patient Samples}

After obtaining approval from the Institutional Review Board (\#211612-4) of Rhode Island Hospital, consecutive patients who underwent colectomy were identified. Patient demographics and clinical data were collected after discharge from the hospital. Colon samples were retrieved from the pathology department at Rhode Island Hospital retrospectively during 2004-2009. From each patient, a section was analyzed from the area of abnormality (site of inflammation, perforation, and so on) and from the resected normal margin. The healthy section of the sample served as each patient's own control. Eleven patients who underwent colon resection were identified. There were no differences between the septic and nonseptic groups related to age (55.9 years versus 53.7 years; $p=0.8$ ) or gender (female, $60 \%$ versus male, $67 \% ; p=1$ ). Five patients were assigned to the septic group, three due to Clostridium difficile colitis and two due to acute diverticulitis. Six patients were assigned to the nonseptic group; four of these patients had an elective resection for diverticular disease, one had a resection for chronic ulcerative colitis and one had a resection immediately after perforation during colonoscopy. Patients were grouped according to the indication for colon resection and the pathology report.

The sections were processed by investigators blinded to the clinical data. The $5 \mu \mathrm{m}$ sections were prepared on slides, deparaffinized, rehydrated, antigen retrieved, blocked and incubated with
anti-PD-L1 antibody (clone MIH1, eBioscience) at $4^{\circ} \mathrm{C}$ overnight. The slides were incubated with a biotinylated secondary antibody, followed by a streptavidin-biotin peroxidase and diaminobenzidine to visualize PD-L1 staining (19). Four to six grayscale images were acquired per specimen with a Nikon E800 microscope using a $20 \times$ PlanApo objective and a Spot RT3 camera. Image processing and analysis was performed using ImageJ analysis software. Positive staining was defined through intensity thresholding, and mean intensity measurements were recorded.

\section{Animals}

Male C57BL/ 6 wild-type (WT) mice (8-10 wks [which typically includes a 2-3 wk acclimation period in our animal facility]) were purchased from Jackson Laboratories. PD-L1 ${ }^{-/}$mice were obtained from Dr. L. Chen (Yale University) and maintained in our animal facility. All mice were housed in a temperature and light cycle controlled room. It should also be noted that male mice were chosen for use here, as prior studies stratifying females by estrous state indicated that their survival of cecal ligation and puncture (CLP) was typically better than males $(20,21)$; therefore, in these initial studies examining the impact of PD-L1 gene expression on septic mouse gastrointestinal function, and in the interest of retaining scientific consistency with our prior study of Huang et al. (12), we chose to move this study forward with male mouse subjects only. Experiments were performed in accordance with National Institutes of Health guidelines and with approval from the Animal Use Committee (\#0228-13) of Rhode Island Hospital.

\section{Sepsis Model of Cecal Ligation and Puncture}

Mice were subjected to CLP as described previously (19). In brief, mice were anesthetized and a $1 \mathrm{~cm}$ midline abdominal incision was made aseptically. The cecum was isolated, ligated with 4-0 silk below the iliac valve, punctured 
twice with a 22-gauge needle, gently squeezed to extract a small amount of fecal contents and returned to the abdomen, which was then closed in two layers. Mice were resuscitated with $1 \mathrm{~mL}$ Ringer's lactate solution subcutaneously. Sham mice were treated identically, except that the cecum was neither ligated nor punctured. With the approval of the Institutional Review Board (\#0228-13), both pain medications and antibiotics were withheld based on scientific concerns that these might initially act as immune response confounders for the proposed experiments $(22,23)$.

\section{Isolation of Mouse Intestinal Epithelial Cells}

Mouse intestinal epithelial cells (IECs) were isolated as previously described, with minor modification (24). Mice were killed $24 \mathrm{~h}$ post-CLP, and the small intestine was dissected/washed in Hank's balanced salt solution (HBSS) containing $5 \%$ fetal bovine serum (FBS) (Life Technologies). The Peyer's patches, fat and mesentery were removed, and the intestine was slit open, rinsed and cut into $1 \mathrm{~cm}$ segments. The segments were incubated in a shaker for $90 \mathrm{~min}$ at $37^{\circ} \mathrm{C}$ in three changes of $25 \mathrm{ml} \mathrm{HBSS} / \mathrm{FBS}$ containing $1 \mathrm{mM}$ dithiothreitol/0.5mM EDTA (Sigma). The cell suspension from three incubations were pooled, centrifuged, resuspended in HBSS/FBS and filtered through nylon wool columns to remove mucus, tissue debris and dead cells. Cells were washed and centrifuged, and the pellet was resuspended in HBSS /FBS, loaded onto a $40 \% / 70 \%$ discontinuous Percoll (GE Healthcare) gradient and centrifuged at $600 \times g$ for $20 \mathrm{~min}$ at $4^{\circ} \mathrm{C}$. Epithelial cells were collected from the upper $40 \%$ Percoll layer, washed and centrifuged for later analysis.

\section{Cell Culture}

The human colonic epithelial cell line (Caco2) was obtained from American Type Culture Collection and maintained in a standard culture medium consisting of Dulbecco's modified Eagle's medium with $1 \%$ L-glutamine, $0.1 \%$ gentamicin, 1\% HEPES and 10\% FBS (all from Life Technologies). Cells were subcultured every three to four days before reaching confluence.

\section{Western Blot Analysis}

Mouse intestinal tissues and cells were homogenized/lysed in lysis buffer, the lysates were collected and protein concentration was determined (Bio-Rad). Protein samples were separated on 10\% Tris-Glycine sodium dodecyl sulfate gels and transferred to polyvinylidene difluoride membranes. Membranes were blocked and incubated with primary anti-mouse PD-L1 (Bio $\times$ Cell), antiZO-1, -occludin and -claudin-1 antibodies at $4^{\circ} \mathrm{C}$ overnight. After washing, membranes were incubated with horseradish peroxidase-conjugated secondary antibodies and proteins were developed by enhanced chemiluminescence. Image densitometry was collected and analyzed with ImageJ software. $\beta$-actin (Life Technologies) was used as a loading control (19).

\section{Real-Time Quantitative Polymerase Chain Reaction}

Total RNA preparation from cells using the TRIzol reagent and real-time quantitative polymerase chain reaction (QPCR) using the SYBR Green master mix were performed following the manufacturer's instruction (Life Technologies). The RNA concentration was measured, and $1 \mu \mathrm{g}$ RNA was reverse-transcribed with a cDNA synthesis kit. Real-time QPCR was performed to amplify the cDNA for PD-L1 and GAPDH. The primers used are as follows: human PD-L1, sense: 5'-GGTGAGGATGGTTCTAC ACAG-3' and anti-sense: 5'-GAGAACT GCATGAGGTTGC-3'; human GAPDH, sense: 5'-GTGAAGGTCGGAGTCAACG-3' and anti-sense: $5^{\prime}$-TGAGGTCAATGAA GGGGTC-3'; mouse PD-L1, sense: 5'TGCCTTCCAACTCTCGTCTTG-3' and anti-sense: $5^{\prime}$-CAGCGAGCACTTCA TTCAGTC-3'; mouse GAPDH, sense: 5'-TGGCAAAGTGGAGATTGTTGCC-3' and anti-sense: 5'-AAGATGGTGATG
GGCTTCCCG-3' (25). Duplicate

$\mathrm{C}_{\mathrm{T}}$ values were analyzed using the comparative $\mathrm{C}_{\mathrm{T}}(\Delta \Delta \mathrm{Ct})$ method. The amount of target $\left(2^{-\Delta \Delta \mathrm{Ct}}\right)$ was obtained by normalizing to GAPDH relative to a control (nonstimulated cells or sham mice).

\section{Cytokine Determination by ELISA}

Concentrations of TNF- $\alpha$, IL-6, IL-10 and MCP-1 in tissue lysates were measured by ELISA (BD Biosciences) (19).

\section{Flow Cytometry Analysis}

Caco2 cells cultured with and without human recombinant TNF- $\alpha$ (10 ng/mL) and IFN- $\gamma(10 \mathrm{ng} / \mathrm{mL})$ for $24 \mathrm{~h}$ were collected for PD-L1 expression analysis. Cells were incubated with Fc blocker and stained with phycoerythrin (PE)conjugated anti-human PD-L1 (clone 29E.2A3, Biolegend) or isotype control antibodies for $45 \mathrm{~min}$ on ice. Cells were washed and analyzed by BD FACS Array (BD Biosciences) (12). Data were analyzed with FlowJo software.

\section{In Vivo Intestinal Permeability Assay}

Intestinal permeability was measured using an in vivo ligated loop model as described previously (26). Briefly, $24 \mathrm{~h}$ post-CLP, mouse abdomen was reopened, cecum and distal ileum were externalized, an incision was made at $\sim 5 \mathrm{~cm}$ and $\sim 15 \mathrm{~cm}$ (to make a $10 \mathrm{~cm}$ segment of an in situ loop without disrupting the mesenteric vascular arcades and blood supply) proximal to the cecum, and a double-loop ligature was made at both ends of the segment. After flushing the segment with PBS to remove intestinal contents, the distal ligature was tightened. A solution of fluorescein isothiocyanatedextran (FD4, $5 \mathrm{mg}$ in $0.5 \mathrm{ml}$ PBS; Sigma) was injected into the intestinal segment and the proximal ligature was tightened. The gut loop was covered with warm saline-wetted gauze and foil to prevent evaporation and direct light exposure. After $30 \mathrm{~min}$, blood samples were taken from the heart and plasma was collected for analysis. The ileal segment was removed and the length was measured. FD4 concentration in plasma was determined 
by a fluorescent plate reader. Permeability was determined as FD4 $\mathrm{mg} / \mathrm{mL} / \mathrm{cm}$ of intestine.

\section{In Vitro Permeability Assay}

Flux of FD4 across cell monolayer was accessed for paracellular permeability of the epithelial barrier (27). Caco2 cells $\left(3 \times 10^{5}\right.$ cells $/$ well $)$ were plated in six-well transwell plates (Corning) and grown as monolayers for $21 \mathrm{~d}$. Cells were treated in four groups: 1) control group with vehicle, 2) stimulation with recombinant human TNF- $\alpha$ (10ng/mL) plus IFN- $\gamma(10 \mathrm{ng} / \mathrm{mL})$ (concentrations were chosen according to dose response results), 3) TNF- $\alpha /$ IFN- $\gamma$ stimulation with control $\operatorname{IgG}(10 \mu \mathrm{g} / \mathrm{mL})$ pretreatment, and 4) TNF- $\alpha /$ IFN- $\gamma$ stimulation with anti-human PD-L1 antibody $(10 \mu \mathrm{g} / \mathrm{mL})$ pretreatment. PD-L1 antibody, clone 29E.2A3 or control IgG was added to the apical chamber for $1 \mathrm{~h}$ and cytokines were then added to the basal chamber. After 24 h, FD4 in HBSS was added in the apical chamber (final concentration $1 \mathrm{mg} / \mathrm{mL}$ ). Forty-eight hours later, culture media was taken from the basal chambers, fluorescence was determined and FD4 flux was normalized to the negative controls.

\section{Immunofluorescence Microscopy}

Caco2 cells were grown on the cover glass for $3 \mathrm{wks}$ to form a monolayer. After treatment in four groups described above, monolayers were washed gently, fixed, permeabilized and blocked. Monolayers were incubated with anti-ZO-1 or anti-occludin antibodies at $4^{\circ} \mathrm{C}$ overnight. After washing, monolayers were incubated with biotin conjugated anti-rabbit IgG, then Alexa Fluor 546-conjugated streptavidin (Life Technologies). The images were collected with a fluorescent microscope (Nikon Eclipse 80i) using a $20 \times$ objective and a Spot RT3 camera. Three to five images were acquired from cell monolayers for processing and analysis using ImageJ analysis software (19). Positive staining was defined through intensity thresholding, and mean intensity measurements were recorded and data were presented as a percentage of the field.

\section{Presentation of Data and Statistical Analysis}

One way analysis of variance (ANOVA), Mann-Whitney U-test or non-paired $t$ test was used to analyze data. $P \leq 0.05$ was considered statistically significant.

All supplementary materials are available online at www.molmed.org.

\section{RESULTS}

\section{PD-L1 Expression Is Increased in Septic Versus Nonseptic Patients' Colonic Tissue Sections}

Eleven patients who underwent colon resection at Rhode Island Hospital were identified. Five patients were assigned to the sepsis group and six patients were assigned to the nonseptic group (Figure 1A). PD-L1 expression, determined by immunohistochemistry, was markedly elevated in septic versus nonseptic patients' colonic tissue specimens (Figures 1B-D). When comparing the differences of PD-L1 staining (measured in pixels), septic patients had a significantly higher increase in the expression of PD-L1 in the area of abnormality than the normal margin (Figure 1B); however, there were no differences between the abnormal areas and the normal margins in the nonseptic patients (Figure 1C). Therefore, the differences between the two areas within septic patients was significantly greater than within nonseptic patients (non-paired $t$ test; Figure 1D). Figure 1E shows representative images from two of the patients. Panel 1E1 depicts an area of normal margin compared with an area of abnormality from a septic patient (panel 1E2). Panel 1E3 shows normal margin compared with an area of abnormality from a nonseptic patient (panel 1E4).

\section{Sepsis Increases PD-L1 Expression in the Mouse Intestine}

While naïve and/or baseline (presepsis) differences in gut morphology among WT and PD-L1 gene-deficient mice are not different (see Supplementary Figure S2), our previous studies have documented that PD-L1 gene deficiency is associated with decreased morphological evidence of intestinal injury induced by sepsis in mice (12). Initially, PD-L1 expression was assessed in the WT mouse small intestine and colon tissue homogenates after CLP. PD-L1 protein levels were significantly increased in both tissues taken from mice $24 \mathrm{~h}$ postCLP when compared with sham controls (Figure 2A). Studies showed that PD-L1 was detectable in human gastric epithelial cells (17) and colonic epithelial cells $(13,15,18)$. We confirmed that PD-L1 mRNA and protein were constitutively expressed in freshly isolated small intestinal epithelial cells (IECs) from naïve WT mice (Supplementary Figure S1). To determine the temporal nature of PD-L1 expression during the course of sepsis, IECs from the small intestine were isolated at 6, 24 and $48 \mathrm{~h}$ after surgery and PD-L1 expression was assessed. When compared with sham mice, PD-L1 mRNA expression was already increased at $6 \mathrm{~h}$, and this was sustained for 24 and $48 \mathrm{~h}$ after CLP (Figure 2B). While PD-L1 protein expression was not changed at $6 \mathrm{~h}$, it was markedly elevated by 24 and $48 \mathrm{~h}$ post-CLP (Figure $2 \mathrm{C}$ ).

\section{Increased PD-L1 Expression by IECs Correlated with Intestinal Inflammation and Dysfunction after Sepsis}

It has been generally accepted that gut barrier dysfunction, induced by many mediators including cytokines, is a significant contributor to the development of systemic infection and subsequent multiple organ failure seen in critically ill patients (9). To elucidate the role of PD-L1 in the regulation of cytokine production in the ileum following CLP, tissue homogenates from WT or PD-L1 gene-deficient (PD-L1-/-) mice were collected $24 \mathrm{~h}$ after surgery for cytokine analysis. TNF- $\alpha$, IL- 6 and MCP-1 levels in ileum tissues were significantly elevated in septic WT mice compared 
A

\begin{tabular}{|c|c|c|c|}
\hline & Sepsis & Non-Sepsis & p value \\
\hline Age & $55.9(6.5)$ & $53.7(8.7)$ & 0.85 \\
\hline Percent Female & $67 \%$ & $60 \%$ & 1 \\
\hline Area of Abnormality & $46434.8(583.3)$ & $40938.8(3083.3)$ & 0.32 \\
\hline Normal Margin & $40333.1(1036.0)$ & $39933.5(3242.4)$ & 0.86 \\
\hline Difference( $\Delta$ Pixel) & $6101.6(774.1)$ & $1004.7(267.9)$ & 0.001 \\
\hline
\end{tabular}

B

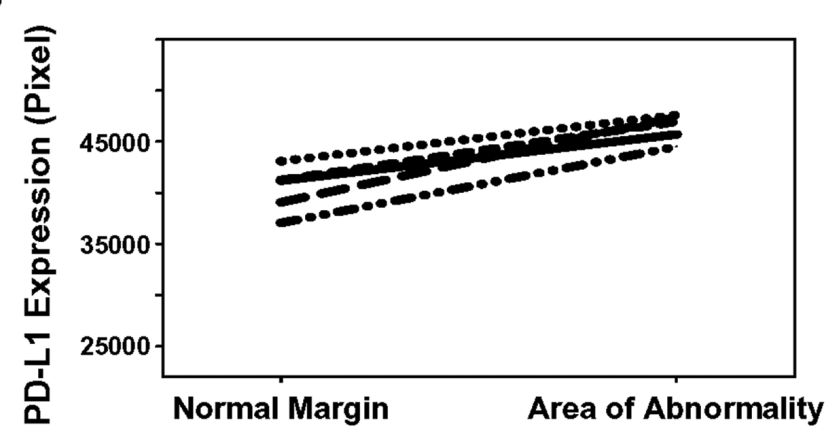

C

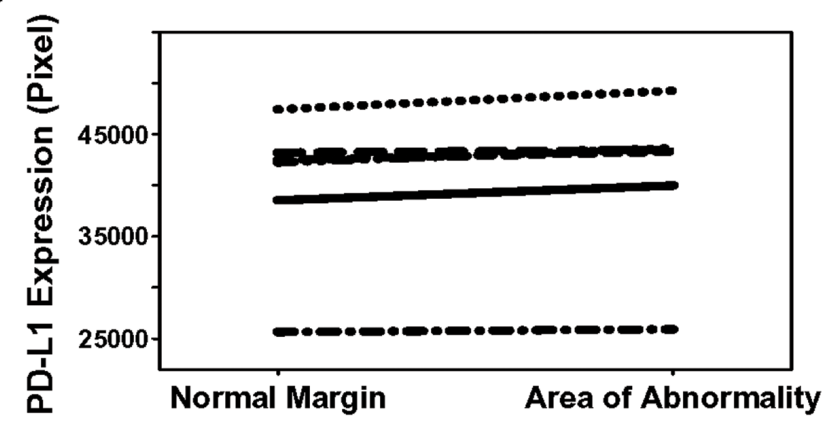

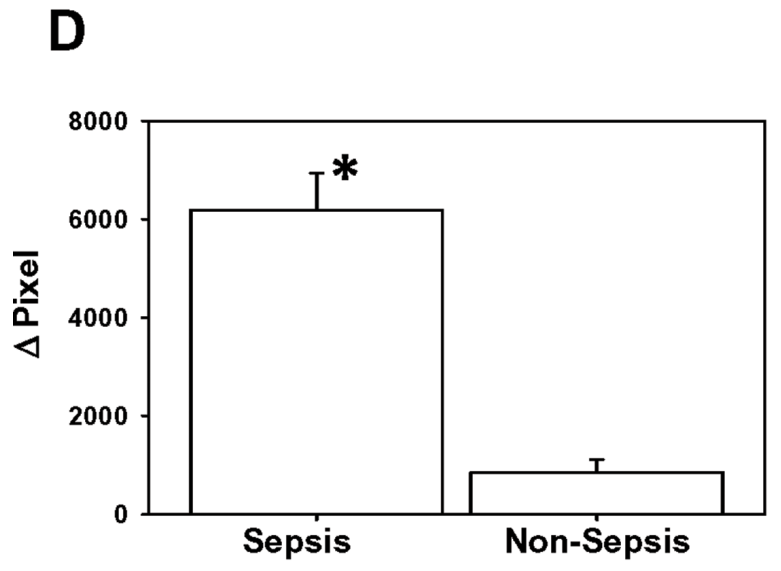

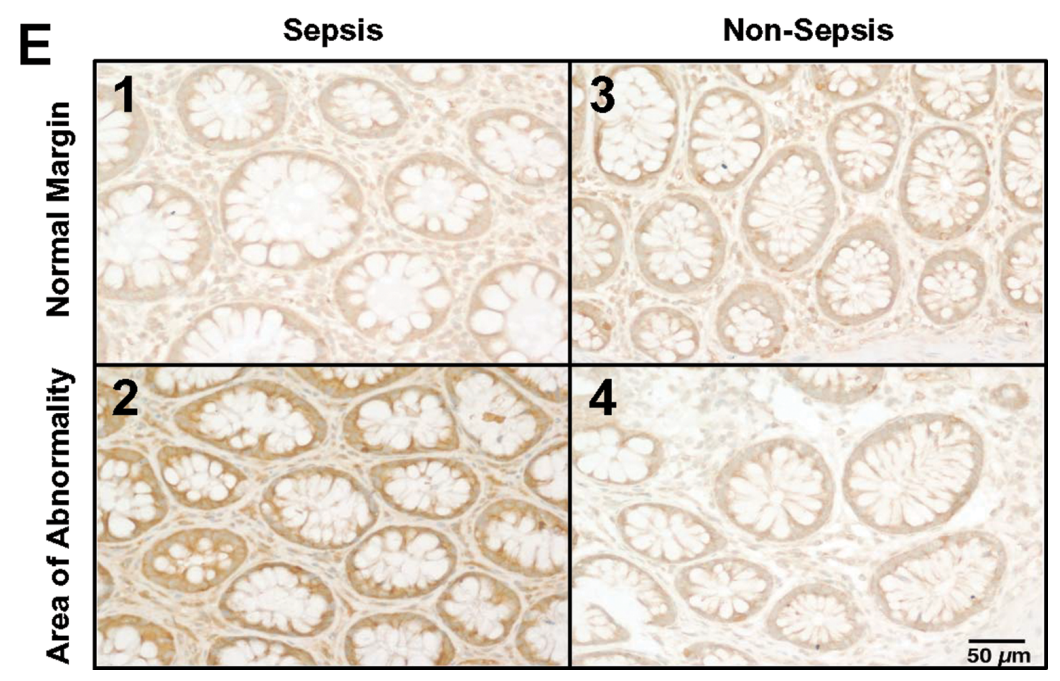

Figure 1. Expression of PD-L1 in colon sections is higher in septic compared with nonseptic ICU patients. Colon histological sections were stained for PD-L1. Four to six grayscale images were acquired per specimen with a Nikon E800 microscope using a $20 \times$ PlanApo objective and a Spot RT3 camera. Positive staining was defined through intensity thresholding, and mean intensity measurements were recorded. Data were expressed as the total area stained on a pixel-by-pixel basis. (A) There was no difference between the septic and nonseptic groups related to age (55.9 years versus 53.7 years; $p=0.8$ ) or gender (female, 60\% versus male, 67\%; $p=1$ ). (B) Significantly higher PD-L1 staining was shown in the area of abnormality compared with the normal margin of septic patients $(n=5)$, while no change in PD-L1 intensity in nonseptic patients was evident $(n=6)(C)$. The calculated extent of change in pixel intensity $(\Delta$ pixel) between the two areas in the same patient were significantly higher in septic versus nonseptic patients $(D),{ }^{*} P<0.05$, nonpaired $t$ test. (E) Representative images of PD-L1 staining from the patients. Panels El and E3 show normal margins from septic and nonseptic patients, respectively. Panel E2 shows the area of abnormality from a septic patient, and panel E4 is the area of abnormality from a nonseptic patient. Original magnifications $\times 200$.

with shams. PD-L1 deficiency blunted the rise of these cytokine levels seen in WT CLP mice (Figure 3A). However, IL-10 levels were not different between all groups.
The tight junction (TJ) is a multiprotein complex and a key regulator of epithelial paracellular permeability. Inflammatory cytokines, such as TNF- $\alpha$ (10) and IL-6 (28), have been shown, under experimental conditions, to disrupt epithelial TJ integrity and compromise epithelial barrier function (29-31). As such, we examined TJ protein levels in WT and PD-L1-/- mice after CLP. We observed 

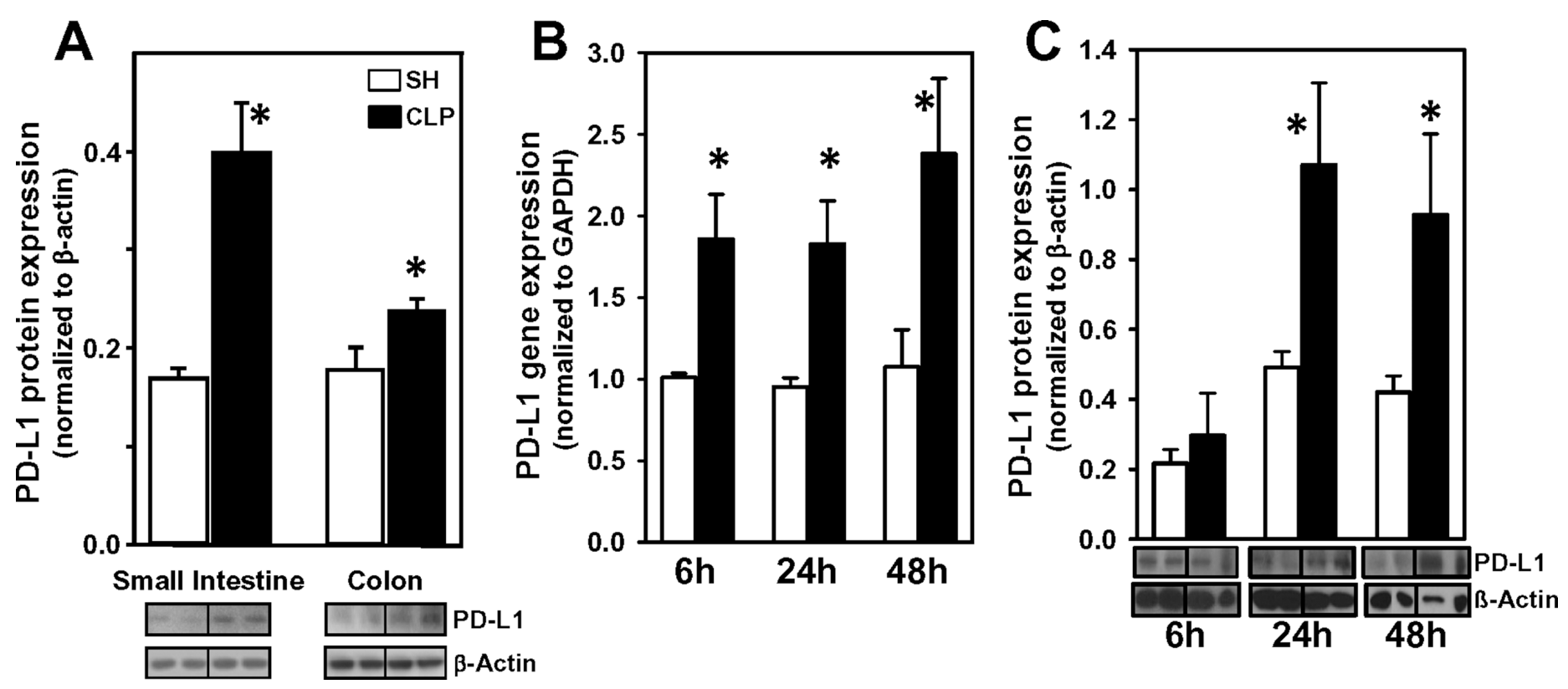

Figure 2. Sepsis increases PD-L1 expression in the mouse intestine. C57BL/6 mice were subjected to sham or CLP. Twenty-four hours after surgery, PD-L1 protein levels in the ileum and colon tissue homogenates were determined by Western blot analyses and semiquantitated by densitometry expressed as integrated density (IDT) values of target protein versus IDT values of $\beta$-actin. (A) CLP induces a significant increase in PD-L1 protein expression in the ileum and colon tissues. Time course of rising gene and protein expression in intestinal epithelial cells (IECs) after CLP. IECs of the small intestine were isolated at 6, 24 and $48 \mathrm{~h}$ after surgery for PD-L 1 expression analysis. (B) PD-L 1 mRNA is significantly increased in the IECs at 6,24 and $48 \mathrm{~h}$ post-CLP compared with sham IECs. (C) While not changed at $6 \mathrm{~h}$, PD-L 1 protein expression is markedly elevated by 24 and $48 \mathrm{~h}$ post-CLP. Significance indicated by ${ }^{*} P<0.05$ versus sham; rank sum test, mean \pm standard error of the mean (SEM); $n=4-8$ mice/group.

a significant reduction in claudin-1, occludin and ZO-1 protein levels in the ileum of WT after CLP compared with sham mice. In contrast, PD-L1 gene deficiency restored claudin-1, occludin and ZO-1 levels in the ileum following CLP (Figure 3B).

Based on the current observation that PD-L1 deficiency reduced sepsis and increased intestinal inflammation and TJ protein loss, we next determined whether PD-L1 expression could mitigate the development of intestinal barrier dysfunction encountered during sepsis in vivo. There was a significant increase in the intestinal epithelial permeability in both WT and PD-L1-/- CLP mice compared with their correspondent shams; however, PD-L1-/- CLP mice had significantly reduced epithelial permeability compared with WT CLP animals (as indicated by lower FD4 concentration in the plasma from intestinal lumen to the circulation) (Figure 3C).

\section{Anti-PD-L1 Antibody Alleviates TNF- $\alpha$ and IFN- $\gamma$ Stimulation-Induced $\mathrm{CacO} 2$ Monolayer Hyperpermeability by Restoring Monolayer Tight Junction Integrity}

In an attempt to translate the mouse in vivo data to humans and better determine if PD-L1 is directly involved in the process of intestinal barrier dysfunction, a human colonic intestinal epithelial Caco2 cell monolayer was established for in vitro experiments. Studies have reported that $\mathrm{Caco} 2$ monolayer barrier dysfunction can be induced by proinflammatory cytokines, such as TNF- $\alpha$ and IFN- $\gamma$, and is associated with the morphological disruption and relocalization of TJ proteins (31-33). With this in mind, we first established the optimum concentration and time of recombinant human TNF- $\alpha$ or IFN- $\gamma$ stimulation for the expression of PD-L1 mRNA in Caco2 cells. Based on the dose-response (Supplementary Figures S3A and S3B) and time-course (Supplementary Figures S3C and $\mathrm{S} 3 \mathrm{D}$ ) results, we confirmed that Caco2 cells could constitutively express PD-L1 protein (by FACS), which was significantly increased following stimulation with TNF- $\alpha$ (10 ng/mL) plus IFN- $\gamma$ (10 ng/mL) (Figure 4A).

To establish that PD-L1 contributes to cytokine-induced hyperpermeability, $\mathrm{Caco} 2$ monolayers grown in the transwell plates were pretreated with anti-PD-L1 or IgG control antibody, then stimulated with TNF- $\alpha /$ IFN- $\gamma$ and permeability determined with the flux of FD4. We found that TNF- $\alpha /$ IFN- $\gamma$ alone and IgG isotype pretreatment followed by TNF- $\alpha /$ IFN- $\gamma$ stimulation significantly increased $\mathrm{Caco} 2$ epithelial monolayer permeability compared with nontreated negative controls. However, anti-PD-L1 antibody treatment prevented the development of Caco2 monolayer hyperpermeability induced by cytokine stimulation (Figure 4B). 

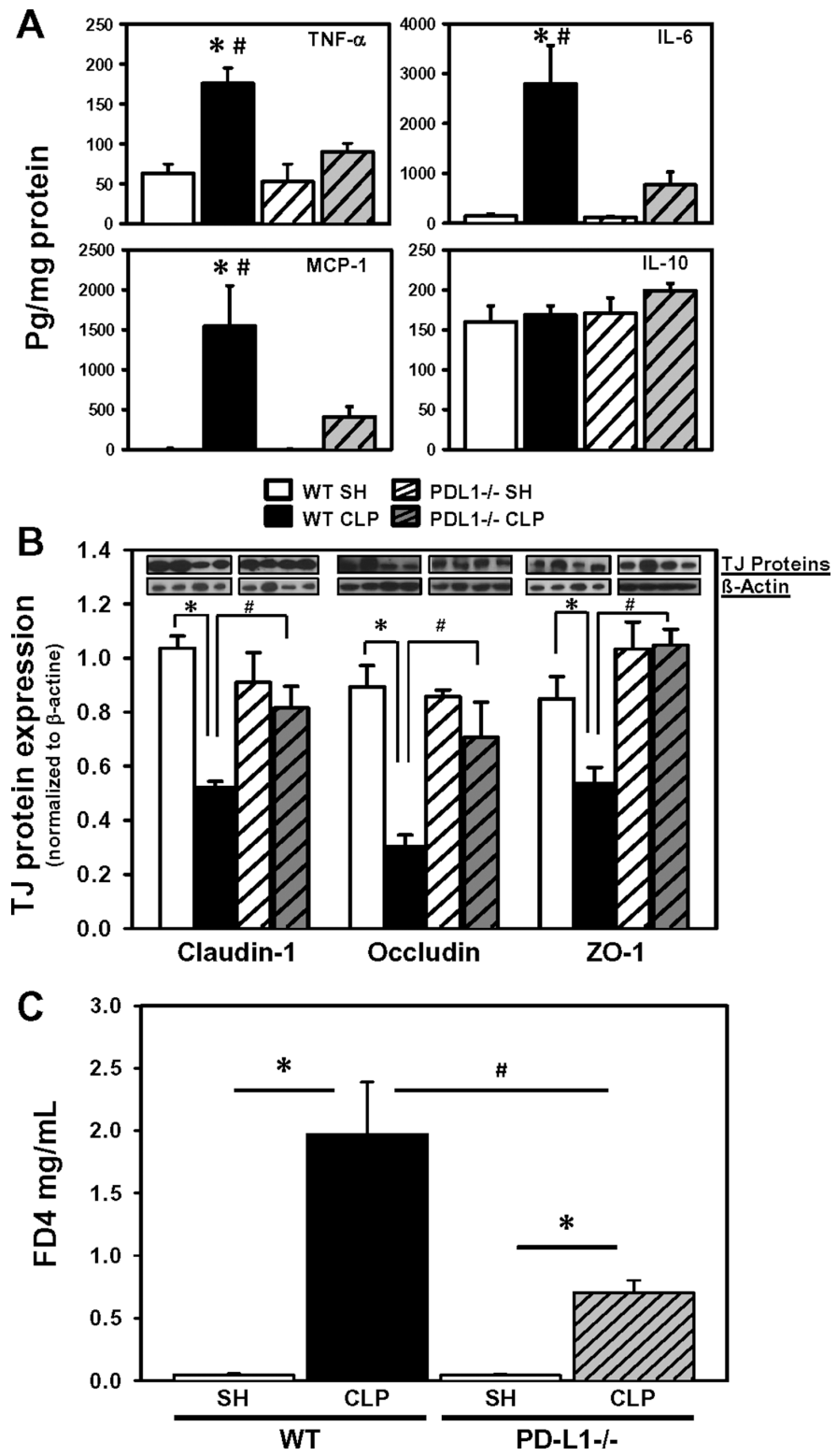

Figure 3. PD-L1 deficiency reduces the sepsis-induced increase in tissue cytokine levels and intestinal permeability while restoring intestinal TJ protein expression following CLP. C57BL/6 and PD-L1 deficient (-/-) mice were subjected to sham or CLP. Twenty-four hours later, the small intestine tissues were harvested for cytokine and TJ protein analysis. (A) Tissue levels of TNF- $\alpha$, MCP- 1 and IL-6 (pg/mL) are increased in septic C57BL/6 mice compared with the shams. However, these cytokine/chemokine levels are significantly decreased in PD-L 1-/- CLP mice compared with septic C57BL/6 mice. IL-10 levels are not changed in all groups. (B) The extent of expression of TJ proteins claudin-1, occludin and ZO- 1 in the ileum is significantly reduced in septic C57BL/6 mice compared with shams. PD-L1 gene deficiency prevents the decline of these proteins following CLP. (C) Intestinal permeability, as an index of intestinal dysfunction, was assessed by a ligated loop model in vivo. Following sepsis, there is a significant increase in the passage of fluorescein isothiocyanate-conjugated dextran (FD4) from the intestinal lumen to the circulatory system in C57BL/6 mice, while PD-L1 gene deficiency markedly reduces sepsis-induced intestinal permeability. ${ }^{*} P<0.05$ versus sham mice; \# $P<0.05$ versus PD-L 1-/- CLP mice. One-way ANOVA and Student-Newman-Keuls test; mean \pm SEM; $n=6-8$ mice/group.
To further investigate the mechanism of PD-L1 in reducing cytokine-induced Caco-2 monolayer barrier dysfunction, the expression of TJ proteins was evaluated by immunofluorescent microscopy. Figure 4C (ZO-1) and 4D (occludin) show that $\mathrm{Caco} 2$ monolayers treated with vehicle, both the TJ proteins ZO-1 and occludin (Figures 4C and D), exhibited consistent staining along the edge of the cells, as expected for negative controls. Treatment with TNF- $\alpha /$ IFN- $\gamma$ alone caused pronounced disorganization and significant reduction in the intensity of ZO-1 and occludin staining (Figures $4 \mathrm{C}$ and $\mathrm{D})$, such that their staining profiles became irregular and discontinuous between adjacent $\mathrm{Caco} 2$ cells. While IgG pretreatment (Figures $4 \mathrm{C}$ and D) also showed similar effects with TNF- $\alpha$ / IFN- $\gamma$ treated alone, anti-PD-L1 antibody pretreatment markedly attenuated the TNF- $\alpha /$ IFN- $\gamma$-induced diffusion/reduction of ZO-1 and occludin staining on Caco2 monolayers (Figures $4 \mathrm{C}$ and D). These data imply that blocking PD-L1 is able to reduce permeability, in part, by preserving TJ architecture in $\mathrm{Caco} 2$ epithelia monolayers after proinflammatory cytokine stimulation.

\section{DISCUSSION}

We have demonstrated that expression of PD-L1 is upregulated in the colons of patients with sepsis, and have also demonstrated the expression pattern and role of coinhibitory molecule PD-L1 in the development of intestinal dysfunction following a polymicrobial septic challenge in mice using the CLP sepsis model. While mouse small intestine IECs constitutively express PD-L1, its expression is significantly increased after sepsis. Using genetic knockout mice, we have found that a deficiency of PD-L1 gene expression appears to contribute to restoration of intestinal barrier function by preventing the cytokine-induced disruption of epithelial TJs during sepsis. In parallel, when the human $\mathrm{Caco} 2$ cell line is used to model the mouse in vivo gut epithelial cell barrier function, we show that PD-L1 blockade is associated 

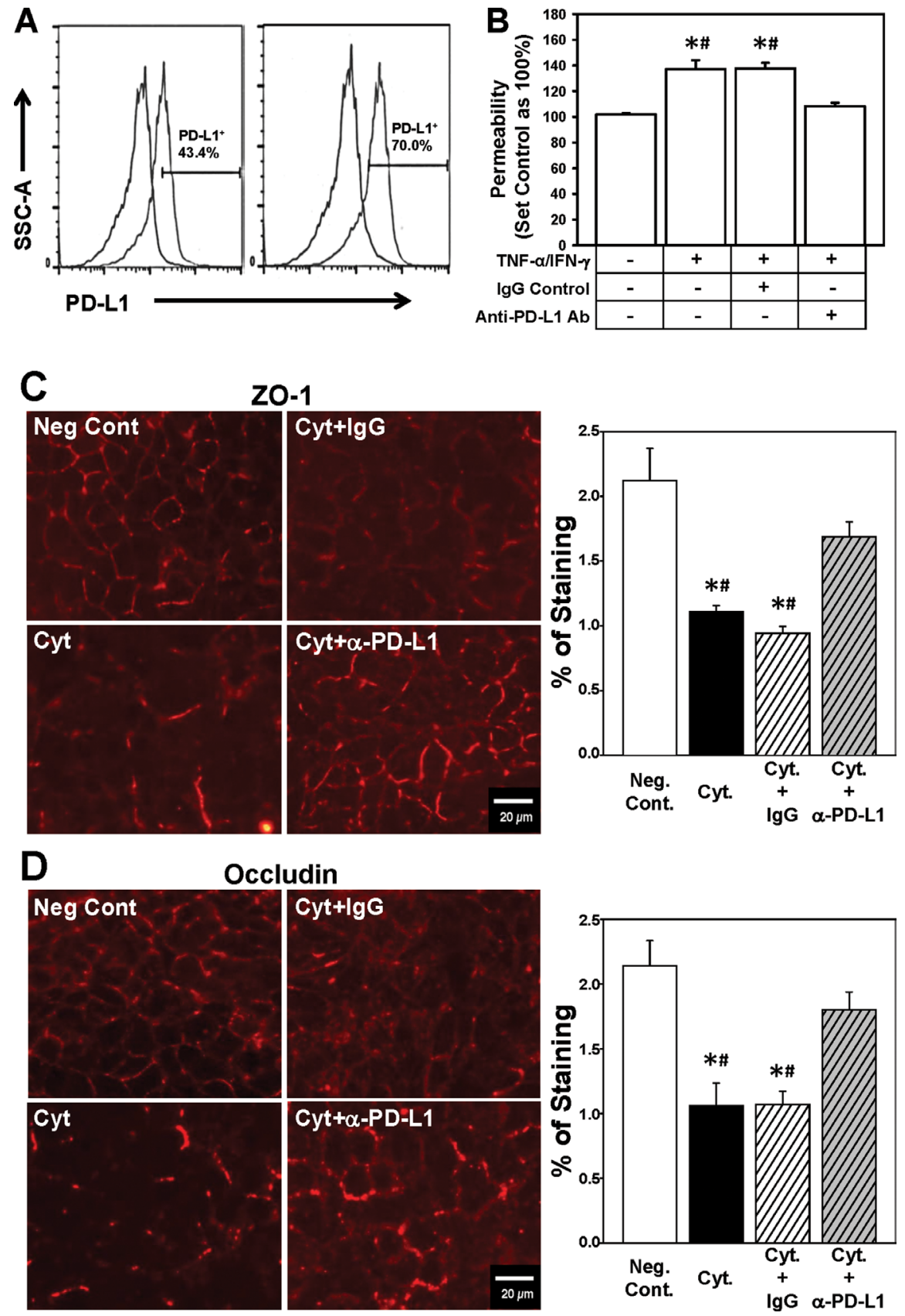

Figure 4. Blockade of PD-L1 prevents TNF- $\alpha /$ IFN- $\gamma$-induced barrier dysfunction of Caco2 monolayer. (A) PD-L1 is constitutively expressed in nontreated Caco2 cells, and human recombinant TNF- $\alpha /$ IFN- $\gamma$ treatment further elevates PD-L1 expression, measured by flow cytometry. To assess the impact of PD-L1 on Caco-2 monolayer barrier dysfunction, cell monolayers were established in transwell plates or glass coverslips for $21 \mathrm{~d}$, pretreated with anti-PD-L 1 or IgG control antibodies, then stimulated with TNF- $\alpha / \mathrm{IFN}-\gamma(10 \mathrm{ng} / \mathrm{mL}$ each). (B) Treatment with TNF- $\alpha /$ IFN- $\gamma$ induces increased monolayer permeability (increased FD4 concentration in basal chambers), while antibody blockade of PD-L1 prevents the effect of cytokines on barrier dysfunction (negative control levels of FD4 was set as 100\%). Data represent the average of three independent experiments, in triplicate for each treatment. ${ }^{*} P<0.05$ versus control; \# $P<0.05$ versus anti-PD-L 1 . Oneway ANOVA and Student-Newman-Keuls test. Representative image of immunofluorescent staining for TJ protein ZO-1 (C) and occludin (D) in Caco-2 monolayers. When compared with negative control (Neg Cont), the monolayers treated with TNF- $\alpha /$ IFN- $\gamma$ (Cyt) showed obvious disruptions in distribution and significantly decreased intensity of ZO-1 (C) and occludin (D) protein staining, calculated in percentage intensity of the fix area. Pretreatment with PD-L1 antibody (Cyt $+\alpha-P D-L 1)$ preserved the integrity of monolayers closer to nontreated control, compared with cytokine treated or lgG + cytokine treated monolayers (C and D). Original magnifications $\times 400$. 
with protecting these monolayers from barrier disruption induced by TNF- $\alpha$ / IFN- $\gamma$ treatment.

Accumulating evidence has demonstrated that the PD-1/PD-L1 pathway plays an important role in regulating the immune-inflammatory response during sepsis, as sepsis can upregulate PD-L1 expression on a variety of immune cells in humans $(2,14)$. In the gastrointestinal tract, human gastric epithelial cells express basal levels of PD-L1, and its expression is significantly increased when exposed to Helicobacter pylori (17). Importantly, the current study is the first to show that expression of PD-L1 is markedly elevated in the colons of patients with sepsis. This association supports the notion that upregulation of PD-L1 in the gut has a potential role in the human septic condition. In keeping with several other studies, increased PD-L1 expression is associated with intestinal dysfunction in patients with inflammatory bowel disease $(13,15,18)$. Furthermore, critically injured or septic patients express significantly increased levels of PD-1 and PD-L1 on their peripheral blood leukocytes $(7,12,34,35)$, which appears to be not only associated with severe injury and/or development of septic shock, but also correlated with mortality in these patients. However, whether this is the cause or effect of the development of the septic state cannot be determined from an observational study.

Recent studies have reported that PD-L1 expression also increased in mice with sepsis induced by CLP $(12,36)$. Additionally, blockade of PD-L1 by antibody suppressed intestinal inflammation and prevented the development of colitis in mice (13), suggesting that PD-L1 may have an important role in regulating the intestinal inflammatory response. Our previous studies have shown that PD-L1/- mice exhibited reduced morphological intestinal injury induced by CLP (12). However, the expression and role of PD-L1 in IEC function during sepsis are not known. In this respect, we initially demonstrated that there was an increase in PD-L1 expression (mRNA and protein levels) in small intestine IECs in response to a septic challenge in mice. These results imply that upregulation of PD-L1 expression on IECs during sepsis could provide a potentially potent tolerogen/ immune suppressive effect through the PD-1:PD-L1 pathway, which might produce an environment for infection controlled by local intestinal immune cells that compromise and affect the prognosis of sepsis.

Studies have indicated that elevated proinflammatory cytokines may play an important role in mediating intestinal barrier dysfunction in sepsis and other inflammatory intestinal diseases $(10,37)$ and in causing disruption of intestinal TJs (38). Here, we showed that TNF- $\alpha$, IL-6 and MCP-1 levels in local ileum tissues were significantly elevated in septic WT mice. PD-L1 gene deficiency blunted the rise of these cytokine/chemokine levels after CLP. Although it is not known if MCP-1 impacts intestinal epithelial function, studies have shown that MCP-1 is involved in endothelial cell dysfunction in various diseases (39). Interestingly, mucosal tissue levels of IL-10 were not changed between all groups in our study, and this was consistent with other reports (40). Since PD-L1 is involved in reducing intestinal inflammation after sepsis, we further determined if PD-L1 deficiency could decrease sepsis-induced intestinal permeability in mice. Consistent with other reports (41-43), intestinal permeability was significantly increased in WT mice after sepsis; however, PD-L1-/- septic mice exhibited decreased ileal permeability compared with WT septic mice. To investigate the cause of sepsisinduced intestinal barrier dysfunction, the effect of PD-L1 on CLP-induced changes of TJ protein expression in the ileum was examined. Compared to WT mice, PD-L1 gene deficiency preserved TJ protein (claudin-1, occludin and ZO-1) levels in the small intestine after sepsis. These results are similar to studies published by Yoseph et al. (44) showing that increased intestinal permeability was associated with changes in TJ proteins at $12 \mathrm{~h}$ after CLP in mice. They demonstrated that sepsis induced a significant increase in claudin-2 and JAM-A and a decrease in occludin and claudin-5, with a trending decrease in claudin-1 and ZO-1. In addition, Li et al. (45) reported that sepsis induced TJ disruption in the colon with diffused and redistributed of claudin-1, $3,4,5$ and 8 , while claudin-2 was markedly increased. In this regard, inflammatory cytokines, induced by experimental sepsis, have been reported to cause intestinal TJ disruption by reducing expression and/or causing the rearrangement of TJ proteins in mice (42). TNF- $\alpha$ has been reported to induce intestinal TJ permeability via myosin light chain kinase activation in animal models and cultured cells $(33,37,46)$. IL-6 has been shown to regulate claudin-2 expression and increase TJ permeability in Caco2 cell monolayers $(40,43)$. Together with our prior observation that PD-L1 gene deficiency reduced intestinal injury and mortality seen in WT septic mice (12), these current findings imply that it is the upregulation of PD-L1 expression in IECs during sepsis that leads to inflammatory mediator release and increased intestinal permeability, and this is due, in part, to TJ disruptions.

To further explore how PD-L1 is directly involved in intestinal barrier dysfunction, an in vitro human $\mathrm{Caco} 2$ cell monolayer model was used to examine the processes of barrier function. Similar to primary IECs derived from mice, Caco 2 cells constitutively express PD-L1, which is further increased upon TNF- $\alpha$ / IFN- $\gamma$ stimulation. This is consistent with those changes documented for human gastric epithelial cells and colonic epithelial HT-29 cells $(15,17)$. Furthermore, we demonstrated that blockade of PD-L1 was able to reduce increased permeability induced by cytokines, while it preserved TJ protein architecture in Caco2 monolayers. However, the limitation in an in vitro Caco2 cell culture setting is that the effects of reducing monolayer permeability by blocking PD-L1 using antibody are unlikely through PD-1: PD-L1 engagement, since PD-1 is not supposed to be expressed on $\mathrm{Caco} 2$ cells 
and there are no $\mathrm{T}$ cells in these cultures providing PD-1. While the PD-1:PD-L1 pathway is well established in adaptive immunity during chronic infections and cancer, its role in acute infections is less clear and more complicated (16). Studies have demonstrated that PD-L1 contributes to sepsis-induced organ injury, including intestine, and lethality $(11,12)$. However, the role of IEC-expressed PD-L1 in intestinal inflammation remains unclear. Interestingly, recent studies have shown that in addition to PD-1, CD80 has been identified as a second binding partner for PD-L1 in humans and mice $(47,48)$, and CD80 has also been reported to be expressed on IECs $(47,49)$. Upon stimulation by microbes or other antigens from the lumen, IECs secrete inflammatory mediators, recruit leukocytes and increase PD-L1 expression. It is possible that during sepsis, the local intestinal inflammatory response could be regulated by either PD-1:PD-L1 and/ or CD80:PD-L1 interactions between IECs and other immune cells, including lymphocytes, antigen-presenting cells or nonimmune cells that express PD-1, CD80, or some unknown potential binding partners for PD-L1. The functional roles and mechanisms of these engagements of PD-L1 and other receptors (ligands) between immune and immune cells and/or immune and nonimmune cells in this system remain to be explored.

\section{CONCLUSION}

Our results provide evidence of increased PD-L1 expression not only in gut/IECs during sepsis in an experimental animal model, but also in colonic tissue specimens of septic patients and a human colonic epithelial cell line. These findings point to a novel role of PD-L1 (beyond that of a simple leukocyte tolerogen) in mediating intestinal barrier dysfunction during sepsis. The increased PD-L1 expression on IECs may also promote the translocation of bacteria/ toxin and a local inflammatory response via its actions on IEC TJ protein dissociation, which could disrupt the integrity of epithelial monolayer stability, thereby leading to intestinal barrier dysfunction. Thus, we think these findings have added significantly to our understanding of the possible roles of PD-L1 in intestinal barrier dysfunction in sepsis as well as potentially other pathological conditions of the gut where PD-L1 expression changes, further illustrating how modulation of PD-L1 may be a novel therapeutic target for this devastating condition of critically ill patients.

\section{ACKNOWLEDGMENTS}

The authors would also like to thank Dr. Lijuan Wang, a pathologist, for sample selection and preparation prior to staining of the sections and Ms. Virginia Hovanesian, Core Research Laboratories, Rhode Island Hospital, for her assistance with microscopy and image analysis of the specimens. This study was supported by National Institutes of Health project grants R01 GM046354 and R35 GM118097 (AA) and GM110495 (DSH), a Shock Society Jr. Faculty Fellowship (CSC) and China Scholarship Council Fellowship \#20120658006 (YW).

\section{DISCLOSURE}

The authors declare that they have no competing interests as defined by Molecular Medicine, or other interests that might be perceived to influence the results and discussion reported in this paper.

\section{REFERENCES}

1. Angus DC, van der Poll T. (2013) Severe sepsis and septic shock. New Engl. J. Med. 369:840-51.

2. Boomer JS, et al. (2011) Immunosuppression in Patients Who Die of Sepsis and Multiple Organ Failure. JAMA. 306:2594-605.

3. Heffernan DS, et al. (2012) Failure to normalize lymphopenia following trauma is associated with increased mortality, independent of the leukocytosis pattern. Crit. Care. 16:R12.

4. Monneret G, Venet F, Pachot A, Lepape A. (2007) Monitoring immune dysfunctions in the septic patient: a new skin for the old ceremony. Mol. Med. 14:64-78.

5. Inoue S, et al. (2013) Reduction of immunocompetent $\mathrm{T}$ cells followed by prolonged lymphopenia in severe sepsis in the elderly. Crit. Care Med. 41:810-19.
6. Drewry AM, et al. (2014) Persistent Lymphopenia After Diagnosis of Sepsis Predicts Mortality. Shock. 42:383-91.

7. Hotchkiss RS, Monneret G, Payen D. (2013) Immunosuppression in sepsis: a novel understanding of the disorder and a new therapeutic approach. Lancet Infect. Dis. 13:260-68.

8. Deitch EA. (2001) Role of the gut lymphatic system in multiple organ failure. Curr. Opin. Crit. Care. 7(2):92-98.

9. Fink MP. (2003) Intestinal epithelial hyperpermeability: update on the pathogenesis of gut mucosal barrier dysfunction in critical illness. Curr. Opin. Crit. Care. 9:143-51.

10. Turner JR. (2009) Intestinal mucosal barrier function in health and disease. Nat. Rev. Immunol. 9:799-809.

11. Zhu W, et al. (2013) PD-L1 blockade attenuated sepsis-induced liver injury in a mouse cecal ligation and puncture model. Mediators Inflamm. 2013:361501.

12. Huang $X$, et al. (2014) Identification of B7-H1 as a novel mediator of the innate immune/proinflammatory response as well as a possible myeloid cell prognostic biomarker in sepsis. J. Immunol. 192:1091-99.

13. Kanai T, et al. (2003) Blockade of B7-H1 Suppresses the Development of Chronic Intestinal Inflammation. J. Immunol. 171:4156-63.

14. Chang K, et al. (2014) Targeting the programmed cell death 1: programmed cell death ligand 1 pathway reverses $\mathrm{T}$ cell exhaustion in patients with sepsis. Crit. Care. 18:R3.

15. Nakazawa A, et al. (2004) The expression and function of costimulatory molecules $\mathrm{B} 7 \mathrm{H}$ and B7-H1 on colonic epithelial cells. Gastroenterology. 126:1347-57.

16. Sharpe AH, Wherry EJ, Ahmed R, Freeman GJ. (2007) The function of programmed cell death 1 and its ligands in regulating autoimmunity and infection. Nat. Immunol. 8:239-45.

17. Wu YY, et al. (2010) Increased programmed death-ligand-1 expression in human gastric epithelial cells in Helicobacter pylori infection. Clin. Exp. Immunol. 161:551-59.

18. Scandiuzzi L, et al. (2014) Tissue-expressed B7-H1 critically controls intestinal inflammation. Cell Rep. 6:625-32.

19. Chung CS, et al. (2010) Deficiency of Bid protein reduces sepsis-induced apoptosis and inflammation, while improving septic survival. Shock. 34:150-61.

20. Zellweger R, Wichman MS, Ayala, A, Stein S, DeMaso CM, Chaudry IH. (1997) Females in proestrus state maintain splenic immune functions and tolerate sepsis better than males. Crit. Care Med. 25:106-10.

21. Knöferl MW, et al. (2000) Do female sex steroids adversely or beneficially affect the depressed immune responses in males after traumahemorrhage? Arch. Surg. 135:425-33.

22. Hugunin KMS, Fry C, Shuster K NJ. (2010) Effects of tramadol and buprenorphine on select immunologic factors in a cecal ligation and puncture model. Shock. 34:250-60. 
23. Guerra AS, et al. (2011) Anti-inflammatory and antinociceptive activities of indoleimidazolidine derivatives. Int. Immunopharmacol. 11:1816-22.

24. Chung CS, Xu YX, Wang W, Chaudry IH, Ayala A. (1998) Is Fas Ligand or Endotoxin Responsible for Mucosal Lymphocyte Apoptosis in Sepsis? Arch. Surg. 133:1213-20.

25. Mühlbauer M, et al. (2006) PD-L1 is induced in hepatocytes by viral infection and by interferon-alpha and -gamma and mediates T cell apoptosis. J. Hepatol. 45:520-28.

26. Wang W, Smail N, Wang P, Chaudry IH. (1998) Increased Gut Permeability after Hemorrhage Is Associated with Upregulation of Local and Systemic IL-6. J. Surg. Res. 79:39-46.

27. Sappington PL, et al. (2002) HMGB1 B box increases the permeability of Caco-2 enterocytic monolayers and impairs intestinal barrier function in mice. Gastroenterology. 123:790-802.

28. Suzuki T, Yoshinaga N, Tanabe S. (2011) Interleukin-6 (IL-6) regulates claudin-2 expression and tight junction permeability in intestinal epithelium. J. Biol. Chem. 286:31263-71.

29. Madara JL. (1989) Loosening Tight Junctions. J. Clin. Invest. 83:1089-94.

30. Ma TY, et al. (2004) TNF-a-induced increase in intestinal epithelial tight junction permeability requires NF-kB activation. Am. J. Physiol. Gastrointest. Liver Physiol. 286:G367-76.

31. Bruewer M, et al. (2003) Proinflammatory cytokines disrupt epithelial barrier function by apoptosis-independent mechanisms. J. Immunol. 171:6164-72.

32. Ulluwishewa D, et al. (2011) Regulation of tight junction permeability by intestinal bacteria and dietary components. J. Nutr. 141:769-76.

33. Zolotarevsky Y, et al. (2002) A membranepermeant peptide that inhibits MLC kinase restores barrier function in in vitro models of intestinal disease. Gastroenterology 23:163-72.

34. Guignant C, et al. (2011) Programmed death-1 levels correlate with increased mortality, nosocomial infection and immune dysfunctions in septic shock patients. Crit. Care. 15:R99.

35. Huang X, et al. (2009) PD-1 expression by macrophages plays a pathologic role in altering microbial clearance and the innate inflammatory response to sepsis. Proc. Natl. Acad. Sci. U.S.A. 106:6303-38.

36. Zhang Y, et al. (2010) PD-L1 blockade improves survival in experimental sepsis by inhibiting lymphocyte apoptosis and reversing monocyte dysfunction. Crit. Care. 14:R220.

37. Clayburgh DR, et al. (2005) Epithelial myosin light chain kinase dependent barrier dysfunction mediates $\mathrm{T}$ cell activation induced diarrhea in vivo. J. Clin. Invest. 115:2702-15.

38. Al-Sadi R, Boivin M, Ma T. (2009) Mechanism of cytokine modulation of epithelial tight junction barrier. Front. Biosci. 14:2765-78.

39. Simionescu M. (2007) Implications of early structural-functional changes in the endothelium for vascular disease. Arterioscler. Thromb. Vasc. Biol. 27:266-74.

40. Wang Q, Fang CH, Hasselgren P. (2001) Intestinal permeability is reduced and IL-10 levels are increased in septic IL-6 knockout mice. Am. J. Physiol. Regul. Integr. Comp. Physiol. 281:R1013-23.

41. Wu R, et al. (2009) Orexigenic hormone ghrelin ameliorates gut barrier dysfunction in sepsis in rats. Crit. Care Med. 37:2421-26.

42. Fredenburgh LE, et al. (2011) Cyclooxygenase-2 deficiency leads to intestinal barrier dysfunction and increased mortality during polymicrobial sepsis. J. Immunol. 187:5255-67.

43. Yang J, et al. (2013) Heprim-binding epidermal growth factor-like growth factor improves intestinal barrier function and reduces mortality in a murine model of peritonitis. Surgery. 153:52-62.

44. Yoseph BP, et al. (2016) Mechanisms of intestinal barrier dysfunction in sepsis. Shock. 46:52-59.

45. Li Q, Zhang Q, Wang C, Liu X, Li N LJ. Distruption of tight junctions during polymicrobial sepsis. J. Pathol. 2009;218:210-21.

46. Ye D, Ma I, Ma TY. (2006) Molecular mechanism of tumor necrosis factor-alpha modulation of intestinal epithelial tight junction barrier. Am. J. Physiol. Gastrointest. Liver Physiol. 290:G496-504.

47. Butte MJ, Keir ME, Phamduy TB, Sharpe AH, Freeman GJ. (2007) Programmed death-1 ligand 1 interacts specifically with the B7-1 costimulatory molecule to inhibit $\mathrm{T}$ cell responses. Immunity. 27:111-22.

48. Ghiotto M, et al. (2010) PD-L1 and PD-L2 differ in their molecular mechanisms of interaction with PD-1. Int. Immunol. 22:651-60.

49. Miyauchi E, et al. (2013) Bifidobacterium longum alleviates dextran sulfate sodium-induced colitis by suppressing IL-17A response: involvement of intestinal epithelial costimulatory molecules. PLoS. One. 8:e79735.

Cite this article as: Wu Y, et al. (2016) A Novel role for programmed cell death receptor ligand-1 in sepsis-induced intestinal dysfunction. Mol. Med. 22:830-40. 